\section{HACIA UNA REGENERACIÓN URBANA 2.0. BARCELONA COMO REFERENCIA}

Dr. Arq. Francesc Peremiquel Lluch ${ }^{1}$

\section{Resumen}

El artículo trata sobre la regeneración urbana llevada a cabo durante los últimos cuarenta años, poniendo en valor los métodos e instrumentos de la práctica urbanística que responden a este objetivo, con distintos nombres, pero misma finalidad. Esto, a partir de los estudios realizados en diversas tesis doctorales sobre la experiencia de Barcelona y su entorno. Se distinguen dos periodos que forman parte de una misma manera de hacer y tienen un similar instrumental de actuación: el del regreso a la ciudad -basado en la urbanidad como objetivo central- y el de la sostenibilidad como principio

\section{TOWARDS A 2.0 URBAN REGENERATION. BARCELONA AS A REFERENCE}

Dr. Arq. Francesc Peremiquel Lluch

\section{Abstract}

This article focuses on the urban regeneration carried out during the last forty years. It is based on the experience of Barcelona and its surroundings, highlighting the methods and instruments used in urban practice. In most cases, with different names but with equivalent purposes, it responds implicitly to this objective. It can be distinguished two periods that have a similar way of doing, using similar instruments of intervention. One is based on the return of the city and the other on the sustainability as development basic principle. This critical appraisal allows us to define somes pecific 
básico de desarrollo. La valoración crítica permite definir algunos elementos concretos a considerar en el programa del proyecto urbano futuro, tanto en términos de estrategia como de intervención táctica, que nos acerca a un modelo renovado de regeneración: la regeneración urbana 2.0.

PALABRAS CLAVE: REGENERACIÓN URBANA, TRANSFORMACIÓN URBANA, PROGRAMA URBANO, PROYECTO URBANO, URBANIDAD.

Recibido: 2020-05-22

Aceptado: 2020-09-11 elements to consider in the future urban project program, both in terms of strategy and tactical intervention. All this brings us closer to a renewed model of regeneration, the urban regeneration 2.0.

KEYWORDS: URBAN REGENERATION, URBAN TRANSFORMATION, URBAN PROGRAM, URBAN PROJECT, URBANITY.

Received: 2020-05-22

Accepted: 2020-09-11

1 Departamento de Urbanismo y Ordenación del Territorio, Escuela Técnica Superior Arquitectura de Barcelona, Universidad Politécnica de Catalunya, España https://orcid.org/0000-0002-2902-3658. Correo electrónico: peremiquel@coac.net 


\section{Introducción}

Desde finales de los años 70 en la ciudad europea se plantea la cuestión de la regeneración urbana como un tema central de la discusión urbanística. Surge como una necesidad derivada del reconocimiento, después de largos periodos de expansión urbana, de la necesidad de volver la mirada sobre la ciudad construida para revertir sus importantes déficits y deficiencias actuales. Para ello se plantean de nuevo, tanto los objetivos, programas e instrumentos de análisis y proyectación, así como nuevos mecanismos de implementación urbana.

El contexto es la salida de la crisis de los años 6070 (mayo del 68, crisis del petróleo del 73). La motivación es la desconfianza y agotamiento de los mecanismos utilizados en la reconstrucción europea de postguerra (Itriago, 2006; Peremiquel y Sotoca, 2012), particularmente la reconstrucción social mediante el estado de bienestar que no había alcanzado a todos por igual y había dejado situaciones urbanas a corregir. La confianza en la morfología, el proyecto urbano y la gestión son elementos claves de un proyecto considerado de éxito.

Cuarenta años más tarde, parece oportuno hacer balance de este periodo con la voluntad de volver al principio, para apuntar líneas de actuación para escenarios futuros. Desde una constante mirada crítica sobre el periodo, en el entorno de Barcelona y sus alrededores como laboratorio de investigación, y mediante el análisis continúo -plasmado en trabajos académicos que nutren de datos abundantes, evidencias y testimonios de lo sucedido, se plantea una reflexión retrospectiva y un ensayo prospectivo sobre aspectos a abordar en un futuro próximo.

Partiendo de una reflexión semántica sobre el prefijo "re", se analiza el periodo en dos partes, la primera, hasta finales de los 90, la que llamamos regeneración urbana 1.1 y que atiende fundamentalmente al regreso a la ciudad mediante el objetivo de recuperar la urbanidad, y el periodo más reciente, después de la irrupción de la globalización posterior a la caída del muro de Berlín y que introduce el debate de la sostenibilidad, la regeneración urbana 1.2.

Para finalizar se plantea una nueva regeneración urbana; la 2.0, en la que emerge una nueva modernidad basada en la integración de la sostenibilidad en la urbanidad: Una nueva urbanidad sostenible que es necesario re-programar.

\section{Una cuestión semántica}

Habitar, habilitar, utilizar, usar, vivir, generar, fundar, componer, programar, estructurar, coser, hacer... verbos que implican acción y, en infinitivo, marcan objetivos. Son utilizados habitualmente en el debate urbanístico sobre 
los problemas de la ciudad, de los fragmentos urbanos y de los territorios urbanizados.

$\mathrm{RE}$, es un prefijo que unido a un verbo en infinitivo implica volver otra vez a formular este objetivo, lo que presupone un posicionamiento de lo que hay que hacer, pero también la puesta en valor de una cualidad previa. No se plantea algo nuevo, sino una repetición. De lo que se trata es de poner al día, mejorar o adecuar las prestaciones urbanas preexistentes a las necesidades contemporáneas. En definitiva, actualizar funcionalidades y atributos urbanos a las demandas actuales. Esto nos lleva a preguntarnos ¿cuáles son las prestaciones que la ciudad debe ofrecer hoy? y ¿cuáles de éstas son las mínimas imprescindibles? Y si la ciudad actual no las satisface, proponer mejoras para alcanzar los nuevos estándares u objetivos deseables. En base a la respuesta establecer un programa de acción.

La palabra regenerar tiene tres acepciones: la primera, volver a poner una cosa deteriorada en buen estado; la segunda abandonar hábitos o conductas reprochables; y la tercera, tratar material desechable para que pueda volver a ser utilizado. Aplicada a las ciudades su utilización comporta reconocer el deterioro, las malas prácticas y considerar la ciudad como material desechable sobre el que hay que actuar. Hablar de regeneración urbana sobre un lugar implica una consideración despectiva sobre el espacio objeto de intervención, predisponiendo así una cierta forma de trato e incidiendo en las personas que lo habitan.

Regenerar significa, por otro lado, voluntad de restablecer algo que existió. Regenerar los tejidos, en términos sanitarios, permite devolverles su condición de utilidad inicial. Significa atención al deterioro y pérdida de atributos, atención a las deformaciones y a las malformaciones, en general sobrevenidas por el paso del tiempo, y que suponen que en un determinado momento gozaron de unos atributos positivos en el pasado que no gozan en el presente. Una visión, por tanto, positiva del estado inicial.

Esta aparente contradicción entre lo negativo y lo positivo de la regeneración llevaría, en términos de estrategia de intervención, a posiciones extremas. Por un lado, la tabula rasa y la recolonización del territorio, es decir a formular un proyecto de nueva planta. Por otro lado, a las intervenciones de acupuntura, cirugía no invasiva y a las prótesis, que mantienen activo el organismo sin transformaciones morfológicas profundas, pero con limitaciones evidentes. Entre estas posiciones extremas existen múltiples gradaciones en la intervención, las cuales no dependerán de una definición objetiva sino de una lectura interpretativa, tanto del estado del objeto en términos materiales, como de los valores intrínsecos de significación cultural, de los retos, oportunidades y limitaciones que ofrece de proyección futura. 
Reconocer que determinados espacios o tejidos no han gozado nunca de atributos positivos parece poco adecuado, por lo que añadir siempre el prefijo "Re" a la acción urbanística se entiende como lo más conveniente. Evita el problema de encarar lo que se tiene entre manos como una ruina física, económica o social, reduciendo así el impacto social que con otras aproximaciones más drásticas se produciría.

Introducir aspectos semánticos no es en ningún modo banal ya que la significación de las palabras va a condicionar las lógicas y actitudes con las que se va a actuar.

Las estrategias de intervención urbana vinculadas al prefijo Re (habilitar, habitar, generar, estructurar) forman parte de una larga cultura sobre la intervención en la ciudad por los poderes públicos. Sin ánimo de precisar, podemos afirmar que esta cuestión se convierte en un tema central a mitad de siglo XIX, cuando los problemas sanitarios (epidemias) y medioambientales (contaminaciones por el humo de las fábricas) y las pésimas condiciones de vida de la clase trabajadora industrial emergente (proletariado) afectan de modo dramático a las ciudades europeas, que han crecido con la industrialización y el desplazamiento de población del campo a la ciudad sin estar preparadas para ello.

La fórmula mayoritariamente utilizada para responder al problema del crecimiento es la expansión de las ciudades e incluso la descentralización mediante la fundación de nuevas ciudades, pero no menos importante es la reforma urbana (reforma: adaptar la forma), que tiene el objetivo de mejorar las condiciones de vida, además de reestructurar (adecuar la estructura) y reorganizar (adaptar las funciones y actividades) de la ciudad existente.

El caso de París es uno de los más conocidos y divulgado, pero existen otros muchos como Berlín, Londres, Barcelona o incluso Nueva York. El programa urbano de Haussmann para París en los años 50 del siglo XIX es utilizado en muchas ciudades. Apertura de nuevas calles y avenidas, mediante percées que han de resolver los problemas de movilidad emergente sirven para generar nuevos tipos de edificios. Estos permitirán alojar a la población en mejores condiciones de habitabilidad y aportarán mejoras a la imagen de la ciudad y el paisaje urbano, con un claro mensaje de modernización y resolución de los problemas de insalubridad, ineficiencia e inequidad de la ciudad.

Sin embargo, con el devenir de los años se fue implantando la idea de que la ciudad vieja era en muchos casos un espacio insalubre, ineficiente e inadecuado, donde, por razones políticas, lo mejor era no intervenir, sino dejarlo a su suerte y abandonarlo. Convertido en foco de degradación y abandono, decadente y deteriorado, algunos arquitectos urgen intervenir para garantizar la supervivencia de las mismas ciudades mediante una nueva arquitectura desvinculada de la calle 
y con dominio del verde (Le Corbusier, 1964; Sert, 1944).

Ciertas reacciones conservacionistas, por su parte, reivindican para estos espacios valores patrimoniales y monumentales y urgen por su conservación, más superficial y aparente que profunda, manteniendo la imagen urbana, pero sin adecuar (y menudo oponiéndose a ello) las condiciones de habitabilidad a las demandas sociales. Frente a la degradación del patrimonio se impone la idea de la necesidad de restauración primero, y rehabilitación después; esto implica un reconocimiento de valor cultural y artístico del soporte que no siempre existe (Ordóñez, 2018).

Ambas estrategias, tanto la del derribo generalizado como la de la conservación absoluta, suponen en la práctica un abandono de la ciudad construida y centran todos los esfuerzos en la expansión de la ciudad hacia la periferia, que cada vez será mayor, más lejana y que también albergará clases sociales más desfavorecidas. A la larga estos serán los nuevos focos de insalubridad, ineficiencia e inequidad.

Con el tiempo, lo que era nuevo envejece, se deteriora, degrada y degenera, incrementando la magnitud del problema tanto en aspectos cuantitativos como cualitativos. La ciudad deficiente, deficitaria y degenerada no es un problema solo de los centros históricos y cascos antiguos, sino que de diversas maneras se extiende por toda la metrópolis, en tejidos tradicionales, de vivienda masiva y en las urbanizaciones marginales; sobre áreas y recintos industriales obsoletos; sobre terrenos e intersticios abandonados, playas ferroviarias inutilizadas, áreas portuarias inservibles o cuarteles desocupados. Muchos lugares, en los que la ciudad no solo pierde el nombre sino también sus atributos. A los pecados originales del desarrollo se añade el deterioro del paso tiempo.

\section{Regeneración urbana 1.0}

Si bien desde mitad del siglo XIX la regeneración urbana está presente en el desarrollo de la ciudad, es en la ciudad contemporánea donde la necesidad de regeneración irrumpe como un tema central de preocupación. El problema ya no es exclusivo de ciertas partes de la ciudad, sino de todas partes, a distintos niveles y de modo generalizado. El declive ha hecho metástasis. Todo es susceptible o está necesitado de regeneración, incluso la ciudad recién construida. Los conjuntos de vivienda masiva de los 60 ya son un problema en los setenta y los grandes asentamientos marginales surgidos el último cuarto del siglo $\mathrm{XX}$, demandan intervención urgente sin tan sólo estar consolidados.

El punto de inflexión, con la toma de conciencia de que la expansión no es la solución ni la alternativa, se puede situar a finales de la 
década de los 70, con una primera idea que es la del "regreso de la ciudad", reconociendo que el patrimonio urbano colectivo, aun sin valor cultural o patrimonial, no puede ser abandonado y que es necesario actuar desde todos los puntos de vista posibles para recuperar el valor de posición del suelo y el potencial de los espacios y elementos urbanos existentes mediante reurbanizaciones, reequipamientos, reestructuraciones, renovaciones, recolonizaciones y rehabilitaciones. Actividades todas ellas que parten del reconocimiento de un hecho urbano previo que habrá que tomar en consideración antes de actuar. La intervención no será sobre un papel en blanco. Serán aquellos lugares de alto valor de posición ambiental, social y económica, y los territorios sensibles, a menudo frágiles, donde habrá que priorizar la intervención.

Tampoco las estrategias a seguir se formularán sobre un papel en blanco, sino que buscan y encuentran su razón de ser en las nuevas dinámicas urbanas y territoriales, productivas y tecnológicas y en las condiciones económicas derivadas de la globalización, así como en las tendencias sociales emergentes, la base para el desarrollo de un programa de actuación que dé contenido a la necesaria recuperación del espacio y justifique no sólo las lógicas morfológicas y proyectuales nuevas sino también los procedimientos de intervención e implementación más innovadores.

Estas políticas de intervención que llevan casi medio siglo de prácticas y experimentaciones generalizadas se pueden considerar, con la crisis de la última década, un periodo conceptualmente cerrado, al que hemos dado en llamar regeneración urbana 1.0 o primera regeneración, susceptible de ser evaluado en aras a relanzar unas nuevas agendas urbanas y con ellas abordar la nueva regeneración, la 2.0, sobre la ciudad del futuro.

En Europa, la nueva Agenda Urbana marca los objetivos deseables para la formulación de un programa nuevo bajo la orientación de la conferencia Hábitat III de Quito sobre "Vivienda y desarrollo urbano sostenible" y no puede sustraerse de esta evaluación crítica ni ignorar las buenas prácticas que han permitido avances urbanos ejemplares. Años de ensayos permiten reconocer formas de desarrollo (modos o maneras, procesos) y formas del desarrollo (morfologías) útiles para la ciudad futura.

Esta primera fase de la regeneración se puede dividir simplificadamente en dos partes: la primera, 1.1, el retorno de la ciudad y la urbanidad y, una segunda parte, 1.2, de la sostenibilidad y la resiliencia. 


\section{REGENERACIÓN URBANA 1.1: URBANIDAD Y RETORNO DE LA CIUDAD}

La situación crítica en los años 70 se convierte en un conjunto de retos y oportunidades. El problema en la ciudad europea, y en Barcelona en particular, es la obsolescencia física y funcional de muchos tejidos residenciales, el incremento de áreas abandonadas, la degradación ambiental de partes centrales y periféricas de la metrópoli, piezas industriales obsoletas e inservibles, los déficits en servicios y dotaciones y las deficiencias urbanas (patologías en la edificación incluidas) en diferentes partes de la ciudad. Lugares todos ellos donde se concentran retos y se ofrecen oportunidades.

Los problemas y retos a abordar son diversos. El primero es de la accesibilidad, y en consecuencia de la movilidad. Muchos lugares céntricos son inaccesibles por carencia de infraestructura, viaria y ferroviaria, pero también por deficiencias de urbanización y por la existencia de barreras sociales por ser lugares estigmatizados. A menudo los barrios bajos están más cerca de lo que parece. Situarlos en el mapa es uno de los primeros objetivos y urbanizarlos y conectarlos adecuadamente una prioridad. El acceso al transporte público es esencial para que muchos de estos lugares dejen de estar estigmatizados y las comunidades sean integradas. No basta con estar cerca, hay que poder llegar. Una política adecuada de transporte público masivo y eficiente es fundamental. Aunque parezca obvio, el metro es clave. También una red de buses eficientes que complemente la cobertura o la mejora de la urbanización creando espacios dignos y seguros para los movimientos peatonales. Además, normalizar los barrios mediante su reconocimiento es otra prioridad.

El segundo problema es el de las dotaciones, el acceso a las prestaciones y servicios urbanos esenciales, y también la actividad económica. Equipamiento y actividad económica (servicios y comercio especialmente) son la fuente de las relaciones sociales. La escuela es la pieza emblemática. Junto a ella, el mercado, el centro de salud, la instalación deportiva o los centros cívicos forman parte de políticas sectoriales para la cualificación de la ciudad. Los servicios a personas y empresa, los pequeños talleres y actividades artesanales, los comercios, etc. -actividades de la iniciativa privada insertadas en los tejidos- son la clave de la integración, el desarrollo social y personal y de fomento de la urbanidad. Mas allá de su utilidad, indiscutible, esta su significación y lo que representan. La calidad de lo colectivo se manifiesta en su arquitectura y su existencia es un símbolo de protección y seguridad.

El tercer problema es el verde. ¡El verde, verde! No el verde como espacio de ocio sino como expresión de la naturaleza en el entorno urbano. Elemento imprescindible para la salubridad ambiental, la salud física y la sanidad mental. Re-naturalizar la ciudad es un reto 
fundamental, cuando se venía de una tradición en que lo que caracterizaba la urbanización era el asfalto y el verde era ornamental y no una necesidad ambiental. El tamaño creciente de las áreas urbanizadas demanda la aproximación de la vegetación y lo que ella comporta, a las áreas más centrales.

Por último, la vivienda, o mejor la casa en términos de Jan Gehl (2006), en su doble dimensión de espacio arquitectónico protegido y entorno próximo de vida cotidiana. El problema no es solo la baja calidad del alojamiento, escaso, anticuado, con patologías constructivas y obsoleto, sino un entorno exterior inhabitable entre los edificios. El problema no es solamente de alojamiento, sino de entender que la vivienda es un elemento esencial e imprescindible para la transformación urbana. El proyecto residencial se plantea como solución y oportunidad y no como problema. No se trata solo de construir vivienda sino de hacer ciudad con la vivienda. La vivienda es la base y el componente imprescindible del buen proyecto urbano. Al fin y al cabo, no hay ciudad sin habitantes y no se puede regenerar la ciudad sin una mejora de las condiciones de vida y entorno que sean atractivas para los ciudadanos, habitantes residentes o los nuevos residentes. Desde el punto de vista de la viabilidad económica y del negocio inmobiliario, a menudo es la pieza clave para la regeneración.
Barcelona, como una más de las ciudades europeas, sufre estos problemas urbanos, que aborda dos niveles complementarios. El primero es la transformación interior, mediante tres tipos de actuaciones: la renovación polígonos de vivienda masiva, derribando y realojando a sus habitantes por causas de patologías edificatorias y deficiencias tipológicas (Martínez, 2013; Peremiquel y Serra, 2012; Peremiquel y Sotoca, 2012); la normalización y regularización de las urbanizaciones marginales, con déficits de urbanización e irregularidades en la construcción (Giraldo y Peremiquel, 2013), y la mejora de tejidos tradicionales, puestos al día mediante reurbanización y mejora de los equipamientos, poniendo en todos los casos el tema de la tenencia como un factor esencial (Busquets, Ferrer y Calvet 1985).

El segundo, mediante operaciones de completamiento, renovaciones de áreas obsoletas y recolonizaciones urbanas (Martins, 2004). Estas operaciones utilizan espacios intersticiales vacíos y los recintos abandonados como oportunidad para la vertebración urbana, la mejora de los estándares urbanísticos, la accesibilidad y superación de las barreras urbanas. La extensión, colonización y articulación de la metrópolis, pasa por proyectos de características y objetivos similares en la periferia lejana o bordes de la ciudad que completan (Gómez, 2011). 
Las operaciones sobre tramas y tejidos se complementan con estrategias sectoriales singulares (territorialización de políticas específicas como educación, sanidad, cultura, etc.) y operaciones estructurales sobre grandes infraestructuras -vías especializadas de alta capacidad, infraestructuras de transporte ferroviario, grandes parques, etc.- (Alcalá, 2004), y un sinfín de pequeñas actuaciones de todo tipo y escala de operaciones de verde, urbanización, equipamiento y vivienda que permiten la reorganización y adaptación funcional de los espacios interiores y son una oportunidad para la propia ciudad, construyendo ciudad en territorios urbanizados y transformando así la primera periferia (Arteaga, 2010).

Con matices, el reconocimiento de problemas comunes y unas estrategias para abordarlos están presentes en la mayoría de las ciudades, grandes medianas y pequeñas, lo que permite establecer esquemas de actuación similares en ciudades diversas. Una vez realizado el diagnóstico y acordado el tratamiento, la pregunta importante no es el qué sino el cómo.

Si bien la homogenización ayuda a efectos taxonómicos, también implica una cierta uniformización en las prácticas urbanísticas y una descontextualización de estas. La irrupción inevitable en las ciudades de los urbanistas globales y la utilización de profesionales mediáticos como instrumento de propaganda no ha ayudado en nada en esta discusión.
La primera apuesta, a principios de los años 80 , fue por el retorno de la ciudad y los valores de la urbanidad. El principio que inspiró la acción de esta fase de regeneración: la urbanidad es el objetivo que se perseguirá mediante el "retorno de la ciudad".

A pesar de que la ciudad tiene problemas de toda índole, y por tanto retos diversos, es común el principio que inspira la respuesta. Hagamos lo que hagamos, sea cual sea el reto que afrontar, hay que volver a la esencia del hecho urbano, volver a plantear la idea de ciudad y de cuáles son los atributos que responden a las demandas de los ciudadanos.

¿Qué es ciudad hoy? ¿Qué elementos favorecen una urbanidad contemporánea? ¿Qué es una calle o una plaza en la actualidad? ¿Qué valores deben ofrecer? Son preguntas que se formulan de nuevo. La recuperación del concepto de urbanidad, de urbanismo urbano; el regreso a los espacios significativos, la recuperación de una identidad ciudadana para las personas, próxima y diversa, en torno a la calle y la casa, a la plaza y el parque, a la escuela y al equipamiento. La confianza en la arquitectura y el diseño van a ser elementos clave del programa urbanístico y es presentes en las políticas y proyectos de muchos gobiernos a todas las escalas.

Los problemas de estructura, composición, ordenación y diseño urbano, como expresión de escalas diversas de abordar un mismo 
objetivo, ocupan el centro de atención durante al menos dos décadas y la confianza en la forma ocupa un papel central para la redención de los males urbanos, al menos aquellos derivados de su configuración física. El proyecto urbano (Solá-Morales, M. 1988) surge como concepto esencial sobre la concepción de la intervención en la ciudad e introduce el debate plan o proyecto, mal entendidos en la discusión como temas contrapuestos, siendo en realidad complementarios.

Barcelona inicia una política pública de regreso de la ciudad que queda plasmada en publicaciones diversas que periódicamente muestran la compleja actividad urbanística municipal a múltiples escalas, muchas de ellas de iniciativa institucional, que sirven para la divulgación, pero también para la discusión, debate y búsqueda de consenso ciudadano. Es esclarecedor de esta iniciativa la publicación en un número monográfico de la revista Arquitecturas Bis, con el título "La vivienda, la escuela y el resto verde" que inicia la divulgación de estas políticas que acabarán teniendo un amplio consenso social. Los efectos y el impacto de estas políticas no solo son la mejora del paisaje urbano, sino también el reequilibrio de las rentas urbanas, la mejora de la cohesión social y el reforzamiento del sentimiento de pertenencia y la identidad urbana. Diversos trabajos de investigación (Alcalá, 2004; Arteaga, 2010; Gómez, 2011; Martins, 2004) reconocen el impacto de estas estrategias sobre la ciudad física y sobre la ciudad social.

\section{REGENERACIÓN URBANA 1.2: SOSTENIBILIDAD Y RESILIENCIA}

El segundo reto emerge al final de la década de los 90, en un mundo totalmente globalizado, que plantea como problema central la preocupación por el deterioro del medio natural, la desigualdad social y la disrupción digital. La sostenibilidad primero, y la resiliencia unos años más tarde, van a venir a ocupar la posición central en el debate, no porque los problemas que aborda sean nuevos, sino porque se ha popularizado la preocupación por el tema y éste ha entrado a formar parte de las agendas urbanas de los gobiernos, de modo similar a las preocupaciones por la ciudad en los setentas, pero de mayor amplitud, ya que no es un problema del viejo mundo occidental, sino que afecta al planeta entero. Nunca la urbanización había alcanzado tanta magnitud ni impacto sobre el medio; nunca había sido tan destructiva, ni había puesto tan en riesgo la civilización. Esta toma de conciencia se va a concretar en conferencias, encuentros, simposios y publicar en declaraciones, cartas y agendas. La sostenibilidad y la resiliencia se van a reivindicar como un criterio central de cualquier acción o iniciativa urbana. La sostenibilidad como principio básico de cualquier desarrollo y la resiliencia como 
capacidad de recuperarse de los impactos que las nuevas dinámicas urbanas y territoriales van a desencadenar.

Aunque parezca que la sostenibilidad es un tema nuevo, es una preocupación que ha estado presente al menos desde la mitad del siglo XIX y que forma parte esencial de los postulados de la modernidad y también del Movimiento Moderno. No podemos, no debemos y no queremos entender la disciplina sin atender estas cuestiones: desde que Cerda escribiera el Tratado General de Urbanización en el siglo XIX, a los contenidos y orientación los congresos CIAM o los manifiestos y declaraciones formuladas a finales de siglo XX, esta es la preocupación central.

La preocupación por un entorno saludable (salud), eficiente (dinero) y solidario (amor) presidirá la discusión e incidirá decisivamente en el programa de proyecto como la primera premisa para tener en cuenta. La resiliencia viene luego, pero seguramente está antes, ya que los desastres naturales, las crisis económicas derivadas y los consecuentes impactos sociales son los que llevan a cuestionar la sostenibilidad del modelo de desarrollo y su impacto en la fragilidad del planeta, como también a la pregunta sobre la capacidad de recuperación frente a impactos de todo tipo. Un tema que al final es tan sencillo como la evaluación de riesgos y coste de las mitigaciones para poner límites a un desarrollo desbocado, de altos costes sociales, económicos y ambientales.

En la práctica, esta preocupación por la sostenibilidad y la resiliencia va a tener pocas consecuenciasurbanísticas, ya que las demandas programáticas de la ciudad sostenible no dejan de ser un programa urbanístico convencional con estándares urbanísticos convencionales, en muchos lugares todavía pendientes de alcanzar. En la ciudad occidental desarrollada lo único nuevo incorporado son preocupaciones sobre la introducción de tecnologías que favorecen temas ambientales (tratamiento del agua, residuos o energía, sistemas de movilidad, etc.) pero no incorporan reflexiones en torno a problemas de estructura, tamaño asentamiento, distancias, tipologías, etc. Más bien, al contrario, justifican el hacinamiento con la ciudad compacta o la hipermovilidad con la multimodalidad, no favoreciendo la ciudad equitativa de ningún modo.

Los programas de políticas urbanas van a poner énfasis en estos aspectos colocando en primer lugar del debate a las personas y con ellas el problema de la toma de decisiones. Esto implica que la participación ciudadana en los procesos urbanos y el establecimiento de unos procedimientos imprescindibles y transparentes de buen gobierno, en los que la concertación urbana como instrumento de gestión ha ido ampliando espacio en el debate 
en la gestión urbanística de cómo llevar a cabo el proyecto (Almeira y Peremiquel, 2018).

La concertación ha ganado adeptos desde los años 80, como apuesta por la integración de actores y agentes, públicos y privados, con intereses comunes y también confrontados, sentados alrededor de una misma mesa, donde no hay más remedio que llegar a acuerdos. Con matices diversos, la concertación se ha impuesto, aunque, por supuesto, es muy mejorable.

Esto va a comportar la introducción de un tema clave en términos conceptuales, pero también morfológicos: la atención sobrevenida por la diversidad. Diversidad, aspecto imprescindible en el equilibrio de cualquier ecosistema, en el urbano y de los agentes intervinientes en la construcción de la ciudad también. En la forma urbana se va a traducir en que los proyectos van a incorporar la diversidad en todos los aspectos: tipológica, funcional, espacial e imagen, pero también de perfiles sociales, de precios, formas de acceso, etc. mediante unos mecanismos de gestión urbanística innovadores, donde el papel de la administración no se limita solo a controlar la legalidad, sino que interviene como un agente económico relevante (sin ser el único ni el mayoritario) gozando de una posición de privilegio y autoridad que se otorga como administrador de lo público.

En segundo lugar, va a irrumpir en el debate la eficiencia, con el objetivo de alcanzar las máximas prestaciones al menor coste, vinculadas al uso de nuevas tecnologías como una pieza clave. La disrupción digital va a ser el instrumento sobre el que se va a plantear una manera distinta de usar la ciudad y la vivienda. Los medios que la tecnología aporta cambian las lógicas de comportamiento, pero sobre todo hacen posible el uso de energías alternativas, materiales innovadores, sistemas de movilidad nuevos, etc. que deberían llevar a pensar el espacio urbano y social de manera distinta.

Las formas de producción también se modifican y con ellas los lugares y modos de vida se deberían adecuar, pero no lo hacen. Barcelona ciudad, por poner un ejemplo, atrae una masa de cuatrocientos mil trabajadores de la región hacia el centro todos los días y una demanda importante de vivienda asociada a esta realidad laboral. Los procesos de movilidad tradicional del centro residencial a la periferia productiva de los años 80 se están convirtiendo en los movimientos actuales desde la periferia residencial al centro productivo. Para evitar esta movilidad, la demanda residencial crece en áreas centrales envejecidas con incrementos de precio desproporcionados y con un impacto ambiental en términos de contaminaciones elevado por esta nueva movilidad hacia el interior y a unos costes en términos de energías personales desproporcionados. La segregación espacial y la exclusión funcional (surgida de la necesidad de adaptar las ciudades a las diversas funciones) con una lógica pragmática y de 
separación de las actividades molestas, nocivas e insalubres en la época de la industrialización, hoy se han convertido en una barrera al desarrollo de la ciudad postindustrial.

Por otra parte, el negocio inmobiliario no tiene límites en sus ambiciones (el edificio más alto, el más caro, el más estrambótico), que las maximiza en el valor de posición. Poner límites a estas ambiciones y levantar las barreras del confinamiento de barrios vulnerables son dos retos fundamentales para un desarrollo urbano saludable y equilibrado.

La sostenibilidad demanda una reflexión en relación con el consumo de energías (las facturables y los esfuerzos personales) y al consumo de materias o recursos, donde proximidad y autosuficiencia se convierten en temas básicos.

En un mundo globalizado, donde la anomia de lugares y el anonimato de la mayoría de las personas, en una sociedad de masas, es una realidad en que cada vez más todo se parece, en la que ha triunfado el estilo "internacional" y la ciudad genérica, en la que da igual el norte o el sur, y lo único importante es la singularidad que satisface el egocentrismo de las estrellas de papel cuché, se hace imprescindible la atención a la identidad que no es la del autor, sino la del objeto y la comunidad en que este se inserta.
La calidad deja de estar asociada a parámetros cuantitativos, para orientarse hacia los aspectos cualitativos, alejándose del tener y acercándose al ser.

Desde un punto de vista conceptual, la resiliencia como principio pone de manifiesto una actitud conservadora que pretende reivindicar un "statu quo", como si éste fuera en sí mismo deseable. A pesar de que éste es un aspecto sobre el cual hay que ser políticamente correcto, parece también necesario denunciar que la resistencia al cambio condena a los desfavorecidos a no tener posibilidad de mejora. La resiliencia es una condición emocional que en la dimensión colectiva tiende a mantener los privilegios, o recuperarlos en caso de impacto, de una parte, de la sociedad que tiene algo que perder. Y efectivamente, todos tenemos algo que perder, pero siempre unos más que otros. En este sentido, la mirada seguramente hay que ponerla más en la resiliencia de las cosas urbanas, su permanencia en el tiempo y la adaptabilidad al cambio, que en las estructuras sociales que albergan.

Estas dos aproximaciones a la regeneración urbana, todavía vigentes, la recuperación de la urbanidad y la sostenibilidad y resiliencia, abren la puerta a reconsiderar aspectos que sin ser nuevos merecen una atención renovada, para continuar progresando. 


\section{Regeneración urbana 2.0. Necesidades y estrategias de intervención: Re- programar el espacio del habitar cotidiano. Actuar}

Las preguntas no han cambiado a lo largo de las décadas o siglos; lo que es imprescindible es adecuar las respuestas a las posibilidades contemporáneas. El reto ambiental, la disrupción digital y la desigualdad social son los tres ejes sobre los que incidir otra vez en la apuesta por un urbanismo redentor; y la confianza en la forma, una vez más en la historia, parece una opción más que razonable como instrumento al servicio del urbanismo para hacerles frente.

Diversidad, aspecto imprescindible en el equilibrio de cualquier ecosistema; sostenibilidad, como componente básico de cualquier desarrollo; e identidad, como característica básica de cualquier asentamiento, son los principios básicos sobre los que debe girar un nuevo programa urbano (Jornet, Llop y Pastor, 2008).

Bajo estos principios de diversidad, sostenibilidad e identidad es imprescindible la definición un nuevo programa urbanístico, reprogramar -volver a definir el programa-, que permita abordar los retos emergentes en el siglo XXI (Peremiquel, 2019).

Inmersos en este debate, en parte metafísico, pero también muy físico, encontramos a faltar mecanismos proyectuales capaces de incorporar respuestas a estas cuestiones que se concreten físicamente y de forma adecuada en la forma urbana, salvo honrosas excepciones. Parece que la forma ya no ofrece mecanismos suficientes para liderar una respuesta. Seguramente, porque los temas de posición, relación, geometría o medida de los elementos urbanos, en un mundo donde imperan los datos, no ocupan la posición que debieran, ni reciben la atención que merecen. Esto es lastimoso, teniendo en cuenta cuanto han aportado a lo largo de la historia en la mejora de las condiciones de vida de las personas, en la eficiencia de las ciudades y en la calidad del medio ambiente. En este sentido, la primera apuesta es por el retorno a la preocupación por la forma.

¿Dónde intervenir? es también una pregunta fundamental. Los territorios sensibles y las periferias vulnerables deberían ser lugares prioritarios para la intervención, tanto por urgencia y necesidad como por oportunidad. Los lugares con vulnerabilidad urbana (áreas urbanas social y físicamente degradadas), periferias frágiles y, de nuevo, la "vivienda" (casa, hogar), deberían ser objeto de intervención preferentes. La periferia hoy ya no 
es un lugar sino una condición, que se produce en posiciones centrales, alejadas del centro o a distancias intermedias. En este contexto, recoser, reciclar y rehabilitar los barrios deben ser objetivos fundamentales (Martínez, 2013).

París, Barcelona, Berlín, Ámsterdam, Tijuana, Bogotá, Quito o Santiago de Chile, entre tantas ciudades del mundo, nos ofrecen ejemplos de situaciones que ejemplifican el dominio de esta condición periférica en el territorio urbanizado. La característica común es la constatación de una situación de déficits cuantitativos que hay que revertir y deficiencias cualitativas que hay que subsanar.

¿Sobre qué intervenir? es la siguiente pregunta. Aunque parezca tópico, continúan siendo temas fundamentales la accesibilidad, física $\mathrm{y}$, de manera muy especial, la metafísica; la actividad social y económica, de servicio, productiva o de ocio; y la habitabilidad, interior y exterior. La clave es crear lugares para vivir y para convivir reconocibles e identificables. Accesibilidad, actividad y habitabilidad con identidad son temas por resolver. Una movilidad alternativa que ponga el peatón en el centro es esencial, donde la proximidad es clave (León, 2019).

Mediante el establecimiento de reglas y parámetros nuevos se debe concretar el cómo intervenir, redefiniendo los estándares urbanísticos y los parámetros regulatorios. Parece razonable que el equilibrio urbano sea un objetivo básico, la ciudad de 50\%: entre público y privado, entre libre y ocupado, entre vehículo propio y transporte público, entre residencia y actividad, entre calzada y acera, entre edificios bajos, medios y altos, entre viviendas grandes y apartamentos reducidos entre géneros, entre edades, entre rentas, etc.

La regeneración urbana necesita de instrumentos de implementación. Más que nunca, una nueva definición de proyectos y planes es necesaria. Pero seguramente no en la lógica jerárquica y estática que conocemos, sino como instrumentos dinámicos capaces de atender a las situaciones cambiantes. Proyectos que anticipen, particularmente la forma mediante la definición de reglas, a través del trazado y la norma y planes que coordinen las acciones, mediante los estándares y los repartos. Instrumentos ambos para el debate donde la reflexividad sea una condición básica de ambos. En muchos lugares, más que regenerar hay que renovar y recolonizar. El proyecto como objetivo y el plan como proceso para ello es clave.

¿Cómo hacerlo? La regeneración urbana necesita de una manera de gestionar distinta. Los proyectos impuestos y autoritarios siempre han encontrado resistencias y al final la realidad se ha revelado. Ningún agente urbano puede actuar hoy con total independencia e impunidad ni por imposición, por lo que para el éxito es clave el consenso, la base para la concertación. 
La ciudad no son los ciudadanos, es el lugar donde habitan los ciudadanos y por tanto es para los ciudadanos y la satisfacción de sus necesidades. Frente a una cultura del urbanismo impuesto desde arriba hacia abajo, se hace imprescindible el planteamiento inverso, de abajo hacia arriba. Un proceso de la participación en la gobernanza y no al revés. Participación que debe ir más allá de la información, la generación de estados de opinión y la simple manipulación. Hay que fomentar el debate inteligente y la confianza en los expertos, donde la ética responsable debe ser una regla fundamental y la transparencia una premisa básica. Las reglas de urbanidad son las normas de comportamiento y de buena educación para vivir en sociedad; la urbanidad, en este sentido, se debe regenerar de nuevo en lo físico y en el comportamiento social también.

La buena vida, la buena forma y la buena educación han de ser los objetivos de la regeneración urbana futura. ACTUAR las acciones concertadas de transformación urbana en áreas residenciales es la expresión de un instrumental útil para abordar la nueva etapa.

\section{A modo de epílogo, la ciudad vacía y la casa llena}

Mientras se estaba escribiendo este texto, el mundo ha empezado a sufrir la pandemia del coronavirus, covid-19. De la noche a la mañana, las ciudades llenas se han quedado vacías, y las casas se han llenado de gente. Las preocupaciones por la congestión, por la hipermovilidad, por el espacio urbano activo, los actos masivos, los grandes eventos... todo se ha convertido en prescindible.

La vida fuera se ha convertido en la vida dentro. No hace falta ir tan lejos, ni tan rápido, ni tanta gente ni todo tan grande. En el confinamiento, la proximidad adquiere un gran valor. Y de repente el tiempo se alarga y las relaciones son distintas. Encerrados empezamos a sentir las limitaciones de una vivienda dormitorio, poco apta para trabajar o aislarse, para hacer deporte o socializar. Ha vuelto el hacinamiento a las casas (para quienes las tienen, pues icómo se va a confinar en casa aquel que no tiene casa?) y la necesidad de un espacio exterior habitable individual para respirar. La necesidad de dispersión por el territorio en comunidades pequeñas y más aisladas (hasta los pueblos más pequeños se cierran a los visitantes casuales por miedo a contaminación), pero seguramente más solidarias; una urbanización distinta para una urbanidad diferente se hace imprescindible.

Pensar en caliente no es bueno. Las emociones no dejan ver claro. Pero repensar de nuevo el espacio para las personas, de la vivienda a la ciudad o viceversa, repensar de nuevo un cambio de paradigma, es una tarea urgente y necesaria. Un nuevo programa urbano se hace imprescindible. 


\section{Referencias bibliográficas}

Alcalá, L. I. (2004). Hacia la asimilación de las vías segregadas en el ámbito urbano: el caso de las Rondas de Dalt y del Litoral en Barcelona. (Tesis doctorado, Universitat Politècnica de Catalunya, Barcelona). Recuperado de http://hdl.handle.net/2117/94272

Almeira, N. y Peremiquel, F. (2018). La concertación urbanística en proyectos urbanos residenciales. En Ciudad y formas urbanas. Perspectivas transversales. Vol. 5 Formas urbanas, planeamiento, proyecto urbano (pp. 33-42). Zaragoza: Universidad Zaragoza. https://doi.org/10.26754/uz.9788417358846

Arteaga, I. C. (2010). Construir ciudad en territorios urbanizados: transformaciones en la primera periferia. (Tesis doctorado, Universitat Politècnica de Catalunya, Barcelona). Recuperado de http://hdl.handle.net/2117/94289

Busquets, J., Ferrer, A. y Calvet, L. (1985). Evaluación de las necesidades de rehabilitación. Madrid: MOPU.

Gehl, J. (2006). La humanización del espacio urbano: la vida social entre los edificios. Barcelona: Reverte.

Giraldo, L. y Peremiquel, F. (2013). 2 barrios, 2 planes, 2 apuestas: recuperación de barrios de urbanización marginal en Barcelona. QRU: Quaderns de Recerca en Urbanisme, (3), 66-85. https://doi.org/10.5821/qru.9567

Gómez, J. C. (2011). El fragmento urbano residencial en la construcción de la metrópoli barcelonesa.
1976-2006. (Tesis doctorado, sin publicar). Universitat Politècnica de Catalunya, Barcelona, España

Itriago, C. T. (2006). Sobre copias, transformaciones y omisiones: la recomposición de ciudades devastadas. (Tesis doctorado en Urbanismo, Universitat Politècnica de Catalunya, Barcelona). Recuperado de http://hdl.handle.net/2117/94280

Jornet, S., Llop, C., y Pastor, J. (2008). Plec de recomanacions per a la redacció dels Plans Directors i les Àrees Residencials Estratègiques. Universitat Politècnica de Catalunya. http://hdl.handle.net/2117/186662

Le Corbusier. (1964). Oeuvre Complète, 1910-1929. Zurich: Éditions d'architecture.

León, M. F. (2019). Una ciudad para el peatón: recorrido, espacio y red. (Tesis doctorado, Universitat Politècnica de Catalunya, Barcelona). Recuperado de http://hdl.handle.net/2117/129267

Martínez, D. (2013). Refer la Barcelona dels barris metropolitans. QRU: Quaderns de Recerca en Urbanisme, (3), 110-125. https://doi.org/10.5821/qru.9558

Martins, A. (2004). Recolonización urbana. Nuevos fragmentos urbanos en las primeras periferias. (Tesis doctorado, Universitat Politècnica de Catalunya, Barcelona). Recuperado de http://hdl.handle.net/2117/94275

Ordóñez, A. (2018). Habitar el casco antiguo: el uso residencial como modo de conservación contemporáneo. (Tesis doctorado, Universitat Politècnica de Catalunya, Barcelona). Recuperado de http://hdl.handle.net/2117/125839 
Peremiquel, F. (2013). Tornant al futur: un altra mirada per la transformació urbana contemporània. QRU: Quaderns de Recerca en Urbanisme, (3), 100-109.https://doi.org/10.5821/qru.9563

Peremiquel, F. (2019). La vivienda de nuevo: indagaciones urbanísticas sobre las tramas residenciales de la ciudad contemporánea. QRU: Quaderns de Recerca en Urbanisme, (9), 100-118. https://doi.org/10.5821/qru.9626

Peremiquel, F. y Serra, P. (2012). Barcelona. 19782010. Renewal processes on Barcelonian mass housing estates. En A. Sotoca (Ed.), After the project: updating mass housing estates. Actualització de polígons residencials (pp 76-95). Barcelona: Universitat Politècnica de Catalunya. Recuperado de http://hdl.handle.net/2099.3/36605

Peremiquel, F. y Sotoca, A. (2012). After the project: Living conditions, urbanity and metropolitan integration. En A. Sotoca (Ed.), After the project: updating mass housing estates. Actualització de poligons residencials (pp 6-25). Barcelona: Universitat Politècnica de Catalunya. Recuperado de http://hdl.handle.net/2099.3/36605

Sert, J. L. (1983). Poden sobreviure les nostres ciutats?: Un ABC dels problemes urbans, anàlisi i solucions. Barcelona: Generalitat de Catalunya, Departament de Politica Territorial i Obres Públiques.

Solá-Morales, M. (1988). La segunda historia del proyecto urbano. Revista UR, (5), 23-29. 


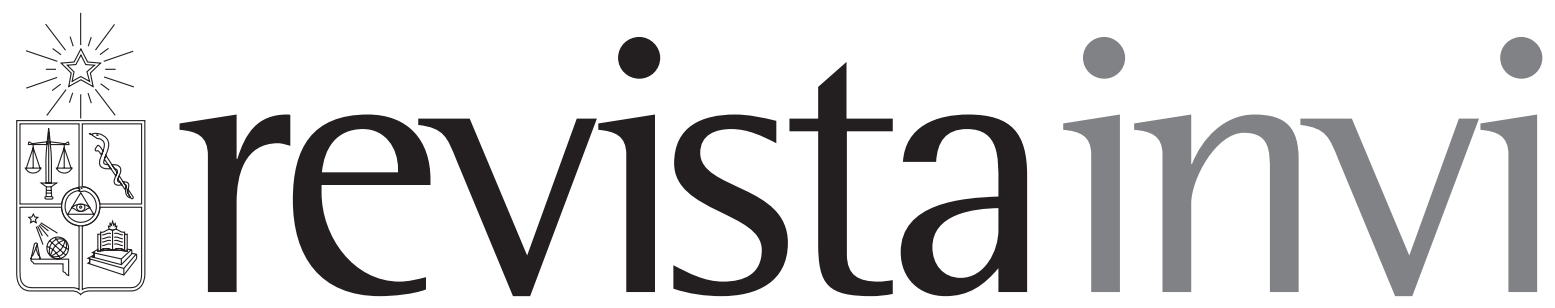

Revista INVI es una publicación periódica, editada por el Instituto de la Vivienda de la Facultad de Arquitectura y Urbanismo de la Universidad de Chile, creada en 1986 con el nombre de Boletín INVI. Es una revista académica con cobertura internacional que difunde los avances en el conocimiento sobre la vivienda, el hábitat residencial, los modos de vida y los estudios territoriales. Revista INVI publica contribuciones originales en español, inglés y portugués, privilegiando aquellas que proponen enfoques inter y multidisciplinares y que son resultado de investigaciones con financiamiento y patrocinio institucional. Se busca, con ello, contribuir al desarrollo del conocimiento científico sobre la vivienda, el hábitat y el territorio y aportar al debate público con publicaciones del más alto nivel académico.

Director: Dr. Ricardo Tapia Zarricueta, Universidad de Chile, Chile.

Editor: Dr. Luis Campos Medina, Universidad de Chile, Chile.

Editor asistente: Dr. Walter Imilan, Universidad de Chile, Chile.

Coeditora: Srta. Sandra Rivera, Universidad de Chile, Chile.

\section{COMITÉ EDITORIAL:}

Dr. Victor Delgadillo, Universidad Autónoma de la Ciudad de México, México.

Dra. María Mercedes Di Virgilio, CONICET/ IIGG, Universidad de Buenos Aires, Argentina.

Dra. Irene Molina, Uppsala Universitet, Suecia.

Dr. Gonzalo Lautaro Ojeda Ledesma, Universidad de Valparaíso, Chile.

Dra. Suzana Pasternak, Universidade de São Paulo, Brasil.

Dr. Javier Ruiz Sánchez, Universidad Politécnica de Madrid, España.

Dra. Elke Schlack Fuhrmann, Pontificia Universidad Católica de Chile, Chile.

Dr. Carlos Alberto Torres Tovar, Universidad Nacional de Colombia, Colombia.

Sitio web: http://www.revistainvi.uchile.cl/

Correo electrónico: revistainvi@uchilefau.cl

Licencia de este artículo: Creative Commons Atribución-Compartirlgual 4.0 Internacional (CC BY-SA 4.0) 